\title{
Long-Term Outcomes of Macular Hole Repair with Triamcinolone Acetonide Visualization
}

This article was published in the following Dove Press journal:

Clinical Ophthalmology

Josh Wallsh (iD

Masumi G Asahi (iD)

Ron Gallemore

Retina Macula Institute and Research Center, Torrance, CA, USA
Correspondence: Ron Gallemore 420 I Torrance Blvd Suite 220, Torrance, CA, 90503

Email rongallemoremd@gmail.com
Purpose: To evaluate the long-term anatomic and visual outcomes of macular hole (MH) repair utilizing triamcinolone acetonide (TA) visualization of the internal limiting membrane (ILM) treated at a tertiary care retina practice.

Methods: Retrospective chart review of eyes undergoing MH repair with ILM peel utilizing TA visualization followed by gas tamponade and facedown positioning between 2014 and 2020. Pre- and post-operative visual acuity (VA), IOP, and anatomic closure based on optical coherence tomography were documented.

Results: Seventy-eight eyes were followed for $2.3 \pm 0.2$ years after primary repair with anatomic closure in $73(94 \%)$ eyes at their final visit and excluding eyes with pathologic myopia, 97\%, and for stage 2 and small MHs, 100\%. In all eyes, VA significantly improved from $0.97 \pm 0.04$ (Snellen: 20/187) to $0.66 \pm 0.06(20 / 91) \operatorname{logMAR}(\mathrm{p}<0.0001)$. There were 16 eyes with 4 years of follow-up, $10(63 \%)$ eyes achieving a VA $\geq 20 / 30$ at that follow-up visit. Stage 2 and $3 \mathrm{MHs}$ had significantly greater improvements in VA than Stage $4 \mathrm{MHs},-0.46 \pm$ 0.06 versus $-0.11 \pm 0.11 \log$ MAR $(\mathrm{p}=0.021)$. Of the $13(17 \%)$ eyes with recurrent MHs, 6 $(46 \%)$ had pathologic myopia and $8(61.5 \%)$ had associated cystoid macular edema. Cataract progression was reported in $52(96 \%)$ phakic eyes and 2 eyes required Ahmed valve placement for management of pre-existing glaucoma.

Conclusion: Long-term results of MH repair with TA for ILM visualization demonstrate that it is safe and effective. Visual acuity continued to improve throughout the follow-up. Pre-existing glaucoma may progress and recurrence is associated with pathologic myopia and macular edema.

Keywords: macular hole, triamcinolone acetonide, internal limiting membrane

\section{Introduction}

Peeling of the internal limiting membrane (ILM) has become a standard surgical procedure in the management of macular holes $(\mathrm{MH}) .{ }^{1}$ Over the years it has become clear that traction from the ILM is a large contributing force in the development of MHs; thus, relieving this traction is paramount to successful closure. To assist with this procedure various dyes have been utilized to improve visualization for more complete removal. ${ }^{2}$

The various dyes used for ILM visualization each come with benefits and weaknesses. Indocyanine green (ICG) and brilliant blue $\mathrm{G}$ (BBG) both provide selective, vibrant staining of the ILM, but with concerns for retinal toxicity associated with both agents. ${ }^{3-8}$ Trypan blue (TB) and triamcinolone acetonide (TA) do not stain the ILM in a traditional sense which may make ILM peeling slightly more complicated. Triamcinolone acetonide appears to have a better safety 
profile than other vital dyes. The use of TA has become commonplace intravitreally both in staining of the posterior hyaloid during vitrectomies and as treatment for macular edema. ${ }^{9,10}$ Although in the past there were concerns for possible retinal toxicity, this was likely due to the preservative, benzyl alcohol. ${ }^{11}$ Preservative-free TA (TRIESENCE, Alcon, Inc., Fort Worth, TX) eliminates this agent and has become the standard formulation for intravitreal TA usage. Choice of dye selection remains a topic for debate with multiple studies comparing different dye choices with various visual acuity results demonstrated. Currently, BBG and ICG are the most popular stains for the ILM, with the 2020 ASRS Preferences and Trends (PAT) Survey reporting $75 \%$ and $16 \%$ of surgeons utilizing each, respectively. In contrast to TA with only $4 \% .{ }^{12}$ Nonetheless, as noted above, there are cases of toxicity and occasional challenges with the other dyes and we have preferred the use of TA for visualizing the ILM. ${ }^{8,13,14}$ Herein, we report our experience with preservative-free TA-assisted $\mathrm{MH}$ repair in both the largest cohort of patients and longest follow-up for such patients.

\section{Methods}

This retrospective interventional case series included all patients who underwent pars plana vitrectomy for $\mathrm{MH}$ with TA-assisted ILM peel between January 2014 and January 2020 with at least 6 months of follow-up. All cases were performed by a single surgeon (R.P.G.). Prior to scheduling of surgery, each patient received a complete ophthalmologic examination including best-corrected visual acuity (BCVA), intraocular pressure (IOP), assessment of lens status, optical coherence tomography (OCT) imaging (Cirrus HD-OCT; Carl Zeiss Meditec; Dublin, $\mathrm{CA}$ ), and, in most cases, fluorescein angiography (FA). Unless complications arose with surgery or in the postoperative period, patients were re-evaluated at least at day one, week one, month one, month two, month six, year one, and bi-annually thereafter.

This study was performed in accordance with the tenants of the Declaration of Helsinki and IRB exempt status was obtained by the Sterling IRB (IRB ID: 8483RPGallemore) on the basis that informed consent was not required for this retrospective study as patient information was to be obtained and recorded in a de-identified manner. Individual health information remained protected, and data confidentiality was maintained throughout the collection of data and writing of this article according to the Health
Insurance Portability and Accountability Act (HIPAA) Privacy Rule and Security Rule.

\section{Procedure}

A standard 23-gauge three port vitrectomy technique was utilized to peel epiretinal membrane and the underlying ILM. After a core vitrectomy, TA (TRIESENCE, Alcon Laboratories Inc., Fort Worth, TX) was injected over the optic nerve and macula and, in the case of stage 2 or 3 MHs, a posterior vitreous detachment (PVD) was created. Additional central vitreous, including the Weiss ring, was removed. After the PVD was created and central vitreous removed, TA was applied to the macula using a 25 -gauge five eighths inch needle bevel down towards the retinal surface. Enough was used to cover the macula from arcade to arcade, then allowed to sit for approximately $10 \mathrm{sec}$ onds, and aspirated off the retinal surface, leaving some granules behind. ILM forceps were then utilized to engage the retinal surface using a pinch, release, pinch and peel technique. Remaining ILM was removed for approximately 1-2 disc diameters surrounding the $\mathrm{MH}$. Periphery was inspected with scleral depression to look for any tears and treated with endolaser if required. Fluidair exchange followed by air-gas exchange was performed utilizing $15 \%$ perfluoropropane (C3F8). Following the procedure, all patients were instructed to perform face down positioning for 1-2 weeks with those eyes with pathologic myopia or history of macular edema recommended to posture for the full 2 weeks.

\section{Study Assessments}

Patients were evaluated for BCVA, IOP, lens status, and MH closure based on findings on OCT at each clinic visit. Each MH was staged using a combination of slit-lamp examination and pre-operative OCT images from the most recent examination prior to surgery. ${ }^{15}$ Additionally, these pre-operative OCT images were used for measurement of MH size based on the narrowest mid-retinal location (small $<250 \mu \mathrm{m}$, medium $250-400 \mu \mathrm{m}$, large $>400$ $\mu \mathrm{m}) .{ }^{16}$ When available duration of $\mathrm{MH}$ was noted with acute MHs considered any of $\leq 6$ months duration and chronic any of $>6$ months duration. Eyes were diagnosed with pathologic myopia based on clinical examination, not refraction, with the presence of myopic degenerative changes including posterior staphyloma. ${ }^{17}$ Notable changes were also commonly seen on OCT images that have been associated with pathologic myopia (Figure 1). ${ }^{18}$ Post-operative complications were noted including $\mathrm{MH}$ 


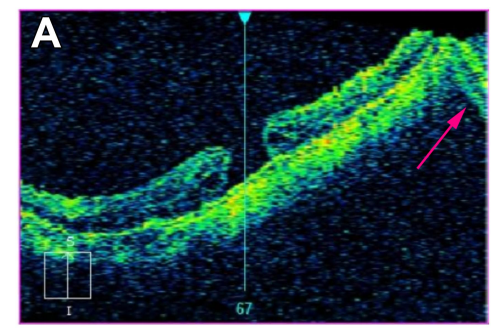

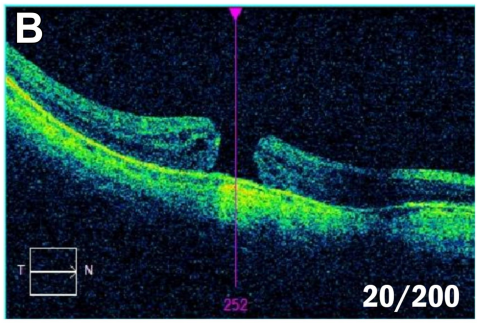

Pre-Operative

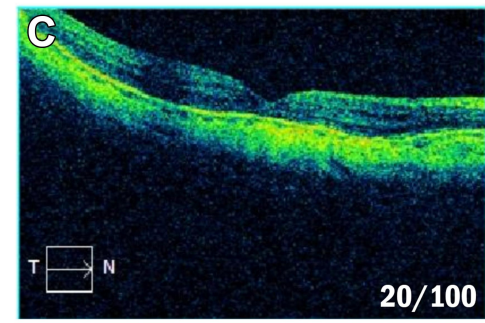

3-months Post-0p

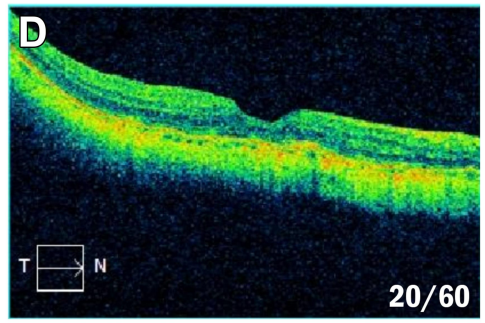

2-years Post-0p

Figure I Optical coherence tomography image of an eye with a macular hole associated with pathologic myopia demonstrating characteristic findings. (A) Pre-operative macular scan oriented vertically from inferior to superior arcade reveals outward bulge of macula associated with posterior staphyloma. This vertical orientation can more clearly demonstrate these findings. Mirror artifact (arrow) is another commonly associated imaging feature in eyes with pathologic myopia. (B) Pre-operative macular scan oriented horizontally (temporal to nasal) demonstrates less pronounced outward bulge and full-thickness macular hole. Post-operative images from (C) 3-months and (D) 2-years showing closed macular hole.

recurrence, retinal detachment, endophthalmitis, etc. Single surgery closure (SSC) was documented as successful $\mathrm{MH}$ closure after the primary operation without recurrence or need for further $\mathrm{MH}$ intervention. Final closure was defined as $\mathrm{MH}$ closure at the final visit with any number of interventions for the $\mathrm{MH}$, including intravitreal C3F8 injection and repeat vitrectomy.

\section{Statistical Analysis}

All data were recorded on Microsoft Excel and statistical analysis was performed using Data Analysis Toolpak software. Snellen visual acuities were converted to the logarithm of the minimum angle of resolution (logMAR) for statistical analysis. For data analysis of pre- and postoperative data, the most recent visit following surgery was used for post-operative value unless otherwise stated. Pre-operative and post-operative BCVA was compared between cohorts utilizing a repeated measures analysis of variance. Closure rates were compared between groups using a Fisher's exact test. Unless specified above, data was analyzed using a paired Student's $t$-test. For all aforementioned analyses, a p-value of $<0.05$ was considered statistically significant.

\section{Results}

Seventy-eight eyes were included that underwent $\mathrm{MH}$ repair with TA-assisted ILM peel during the 6-year study. The majority of eyes (70.5\%) presented acutely within 6 months of symptom development and there was a fairly equal spread of eyes based on $\mathrm{MH}$ stage between 2 (29.4\%), 3 (25.6\%), and 4 (44.8\%) and MH size (Table 1). Eyes were followed for a mean of $2.32 \pm 0.6$ years with a final closure rate of $93.5 \%$ (73 eyes) along with a significant improvement in BCVA. Excluding eyes with pathologic myopia, the SSC rate was $83.5 \%$ and the final closure rate was $97.0 \%$. Eyes with pathologic myopia had an SSC rate of $45.4 \%$ and final closure rate of $72.7 \%$ (Table 2).

Mean visual acuity was noted to have a trend of improvement at each follow-up visit (Figure 2). Of those 16 eyes with 4 years of follow-up, 12 (75.0\%) eyes were noted to have an improvement in BCVA and $10(62.5 \%)$ eyes had BCVA $\geq 20 / 30$. Based on duration of $M H$, the BCVA of eyes presenting acutely improved significantly from $0.85 \pm 0.06$ (Snellen: 20/142) to $0.47 \pm 0.07(20 / 59)$ $\log$ MAR $(\mathrm{p}<0.001)$ and eyes with chronic MHs had a nonsignificant improvement from $1.14 \pm 0.07(20 / 276)$ to $0.98 \pm 0.13(20 / 191) \log \mathrm{MAR}(\mathrm{p}=0.07)$. Comparing the change in BCVA improvement in the acute $\mathrm{MH}$ and chronic $\mathrm{MH}$ cohorts was not significant $(\mathrm{p}=0.15$; Figure 3). The BCVA in stage $2 \mathrm{MHs}$ improved significantly from $0.80 \pm 0.06(20 / 126)$ to $0.36 \pm 0.07(20 / 46)$ $\operatorname{logMAR}(\mathrm{p}<0.001)$, stage 3 MHs improved significantly from $1.06 \pm 0.08(20 / 230)$ to $0.59 \pm 0.10(20 / 78) \log M A R$ 
Table I Demographics

\begin{tabular}{|c|c|}
\hline Patients & 76 \\
\hline Eyes & 78 \\
\hline \multicolumn{2}{|l|}{ Gender } \\
\hline Male: Female & 27: 49 \\
\hline \multicolumn{2}{|l|}{ Age (Years) } \\
\hline Mean \pm S.E.M. & $65.9 \pm 0.9$ \\
\hline \multicolumn{2}{|l|}{ Lens Status } \\
\hline Phakic: Pseudophakic: Aphakic & 53: $24: 1$ \\
\hline \multicolumn{2}{|l|}{ History of Pathologic Myopia } \\
\hline Eyes (\%) & II (13.0\%) \\
\hline \multicolumn{2}{|l|}{ History of Uveitis } \\
\hline Eyes (\%) & I (I.I\%) \\
\hline \multicolumn{2}{|c|}{ Duration of Macular Hole* } \\
\hline \multicolumn{2}{|l|}{ Acute (<6 months) } \\
\hline Eyes (\%) & 48 (70.5\%) \\
\hline \multicolumn{2}{|l|}{ Chronic ( $\geq 6$ months) } \\
\hline Eyes (\%) & $20(29.4 \%)$ \\
\hline \multicolumn{2}{|c|}{ Macular Hole Stage* } \\
\hline \multicolumn{2}{|l|}{2} \\
\hline Eyes (\%) & $23(29.4 \%)$ \\
\hline \multicolumn{2}{|l|}{3} \\
\hline Eyes (\%) & $20(25.6 \%)$ \\
\hline \multicolumn{2}{|l|}{4} \\
\hline Eyes (\%) & 35 (44.8\%) \\
\hline \multicolumn{2}{|c|}{ Macular Hole Size* } \\
\hline \multicolumn{2}{|l|}{ Small $(<200 \mu \mathrm{m})$} \\
\hline Eyes (\%) & $26(33.7 \%)$ \\
\hline \multicolumn{2}{|l|}{ Medium $(200-400 \mu \mathrm{m})$} \\
\hline Eyes (\%) & $26(33.7 \%)$ \\
\hline \multicolumn{2}{|l|}{ Large $(>400 \mu \mathrm{m})$} \\
\hline Eyes (\%) & $25(32.4 \%)$ \\
\hline
\end{tabular}

Note: *Based on eyes with information available for assessment.

$(\mathrm{p}<0.001)$, and stage 4 MHs improved from $1.04 \pm 0.08$ $(20 / 219)$ to $0.90 \pm 0.11(20 / 159) \operatorname{logMAR}(p=0.23)$. Stage 2 and $3 \mathrm{MHs}$ were noted to have significantly greater improvements in BCVA than stage $4 \mathrm{MHs}(\mathrm{p}=$ 0.032 and $p=0.021$; Figure 4). In small- and mediumsized MHs, the BCVA improved significantly from $0.77 \pm$ $0.08(20 / 118)$ to $0.36 \pm 0.07(20 / 46) \log$ MAR $(\mathrm{p}<0.001)$ and $0.97 \pm 0.07(20 / 187)$ to $0.66 \pm 0.10(20 / 91) \log$ MAR $(\mathrm{p}=0.0029)$, respectively. Large MHs had a nonsignificant improvement in BCVA from $1.19 \pm 0.07(20 / 310)$ to 1.02 $\pm 0.13(20 / 209) \operatorname{logMAR}(p=0.19)$. There was no significant difference in the change in BCVA when comparing small, medium and large sized MHs ( $p=0.30$; Figure 5).

The MH closure rates are presented in Figure 6, excluding eyes with pathologic myopia, subdivided based on duration, stage, and size of $\mathrm{MH}$ at presentation. Stage 2 and small MHs were noted to have the highest SSC rate (100\% each). Evaluating final closure rates demonstrated high rates in stage 3 , medium-sized and acutely presenting MHs as well (100\% each). Eyes with stage $4 \mathrm{MHs}$ had an SSC rate of $71.4 \%$ and large MHs had an SSC rate of $72.7 \%$. Single surgery closure rate was significantly greater in both stage 2 and $3 \mathrm{MHs}$ than stage $4(\mathrm{p}=$ 0.027 ) and in small MHs compared to both medium and large MHs $(p=0.042)$. There was no significant difference in the final closure rate between cohorts.

Table 3 presents rates of complications associated with MH repair. The most common complication was cataract progression or surgery in the 54 phakic eyes which occurred in $52(96.3 \%)$ phakic eyes. There were also 2 eyes that required placement of an Ahmed valve for IOP management. Both of these eyes were on maximal tolerated drop therapy for primary open-angle glaucoma prior to MH surgery. One eye developed a retinal detachment over a year after MH surgery which was successfully repaired with vitrectomy, scleral buckle, and silicone oil placement. Prior to retinal detachment development, this patient's BCVA was 20/200 (due in part to exudative macular degeneration) and following eventual silicone oil removal remained 20/200. Recurrent MHs occurred in 13 eyes with a mean of $223.0 \pm 87.6$ days from initial surgery. Eight (61.5\%) eyes recurred within the first 2 months after the initial surgery with the following initial stages: 1 stage 2, 2 - stage 3, and 5 - stage 4 . The stage $2 \mathrm{MH}$ that recurred within the first 2 months had pathologic myopia and was associated with foveoschisis. This $\mathrm{MH}$ remained open despite intravitreal C3F8 placement in the office and a repeat vitrectomy; however, BCVA remained stable at 20/200 throughout. Cystoid macular edema (CME) was noted on OCT in 8 eyes with recurrent MHs due to epiretinal membrane ( 3 eyes), uveitis ( 1 eye), exudative macular degeneration (1 eye), Irvine-Gass ( 1 eye), and idiopathic ( 2 eyes).

\section{Discussion}

We present the largest and longest study of eyes undergoing macular hole repair with triamcinolone acetonide-assisted internal limiting membrane peel. Our cohort of patients include 
Table 2 Outcomes

\begin{tabular}{|c|c|c|c|}
\hline & All Eyes $n=78$ & Eyes with Pathologic Myopia $n=I I$ & Eyes without Pathologic Myopia $n=67$ \\
\hline $\begin{array}{l}\text { Pre-Operative BCVA (logMAR) } \\
\text { Mean } \pm \text { S.E.M. }\end{array}$ & $0.97 \pm 0.04$ & $0.95 \pm 0.13$ & $0.98 \pm 0.05$ \\
\hline $\begin{array}{l}\text { Final Visit BCVA (logMAR) } \\
\text { Mean } \pm \text { S.E.M. }\end{array}$ & $0.66 \pm 0.06$ & $0.84 \pm 0.18$ & $0.63 \pm 0.07$ \\
\hline P-value & $<0.0001$ & 0.64 & $<0.0001$ \\
\hline $\begin{array}{l}\text { Length of Follow-up (Years) } \\
\text { Mean } \pm \text { S.E.M. }\end{array}$ & $2.32 \pm 0.16$ & & \\
\hline \multicolumn{4}{|c|}{ Macular Hole Closure Rates } \\
\hline $\begin{array}{l}\text { Final Closure Rate* } \\
\text { Eyes (\%) }\end{array}$ & $73(93.5 \%)$ & $8(72.7 \%)$ & $65(97.0 \%)$ \\
\hline P-value ${ }^{\dagger}$ & & \multicolumn{2}{|c|}{$<0.05$} \\
\hline $\begin{array}{l}\text { Single Surgery Closure Rate } \\
\text { Eyes (\%) }\end{array}$ & $6 \mathrm{I}(78.2 \%)$ & $5(45.4 \%)$ & $56(83.5 \%)$ \\
\hline P-value ${ }^{\dagger}$ & & \multicolumn{2}{|c|}{$<0.05$} \\
\hline
\end{tabular}

Notes: *Includes eyes closed following primary surgery and those that recurred or failed to close initially and required further intervention. 'Fisher's exact test comparing eyes with and without pathologic myopia.

Abbreviation: BCVA, best corrected visual acuity.

outliers from community retina practices as we represent a tertiary referral center and receive complex cases from retina surgeons. Nonetheless, our results demonstrate comparable rates of macular hole closure, visual acuity improvement, and complications to previous shorter evaluations of triamcinolone acetonide. $^{19-21}$

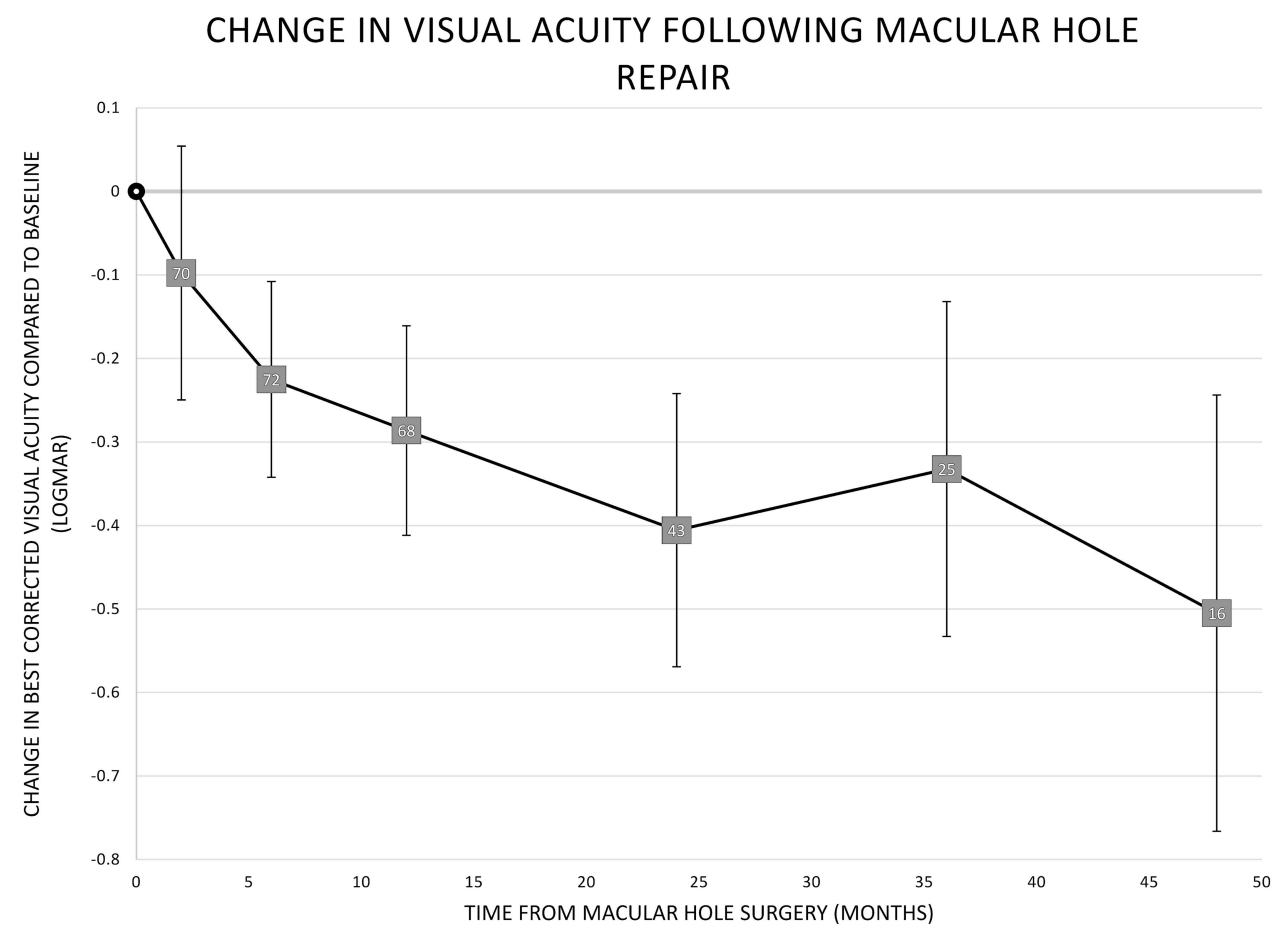

Figure 2 Change in best-corrected visual acuity (BCVA) compared to baseline over time. Negative values indicate a decrease in logMAR which represents improved BCVA. Number of eyes included at each follow-up visit noted next to each data point. Error bars demonstrate $95 \%$ confidence intervals. 


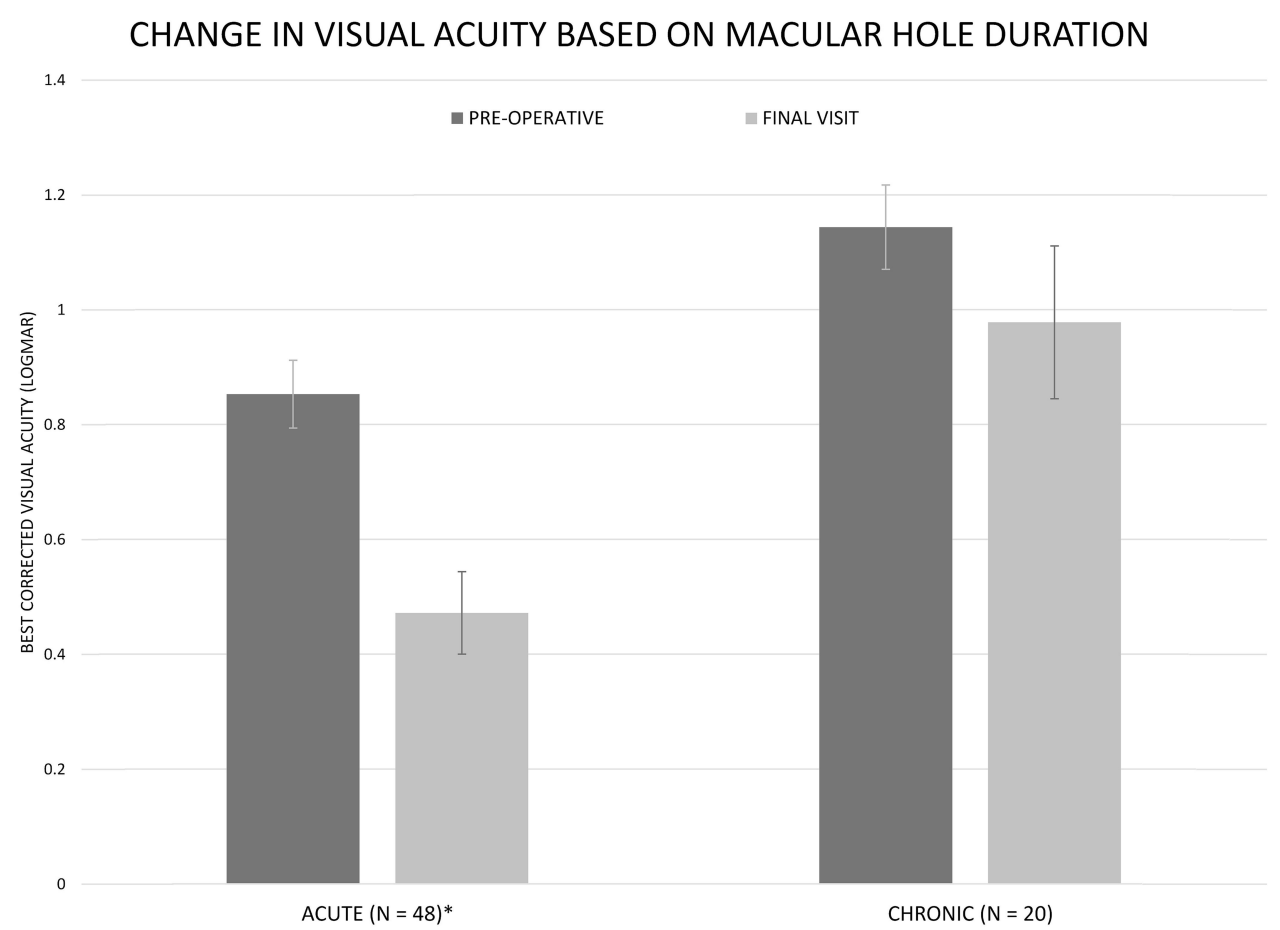

Figure 3 The mean best-corrected visual acuity pre-operatively and at final visit for acute and chronic macular holes. Acute was defined as presenting within 6 months of symptom onset and chronic presented beyond 6 months of symptom onset. Error bars represent standard error of means and $(*)$ indicates statistically significant data $(p<$ $0.05)$.

\section{Closure Rate and Visual Acuity}

Success for MH repair is evaluated based on anatomic closure and BCVA improvement. Previous studies have reported high closure rates between 80 and $100 \%$ with various vital dyes. ${ }^{22}$ This rate is consistent with those rates reported specifically in the setting of TA-assisted

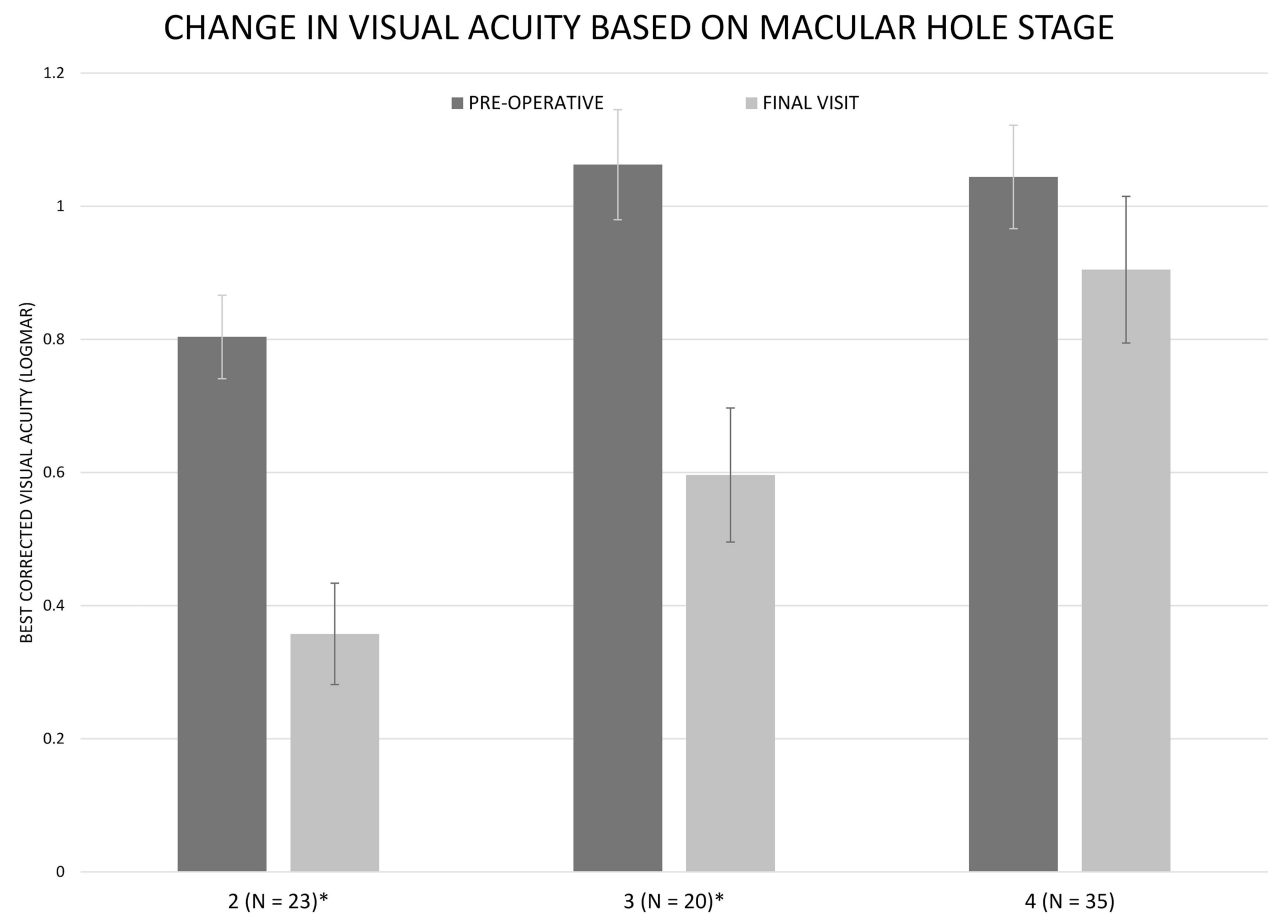

Figure 4 The mean best-corrected visual acuity pre-operatively and at final visit based on stage of macular hole. Error bars represent standard error of means and $(*)$ indicates statistically significant data $(p<0.05)$. 


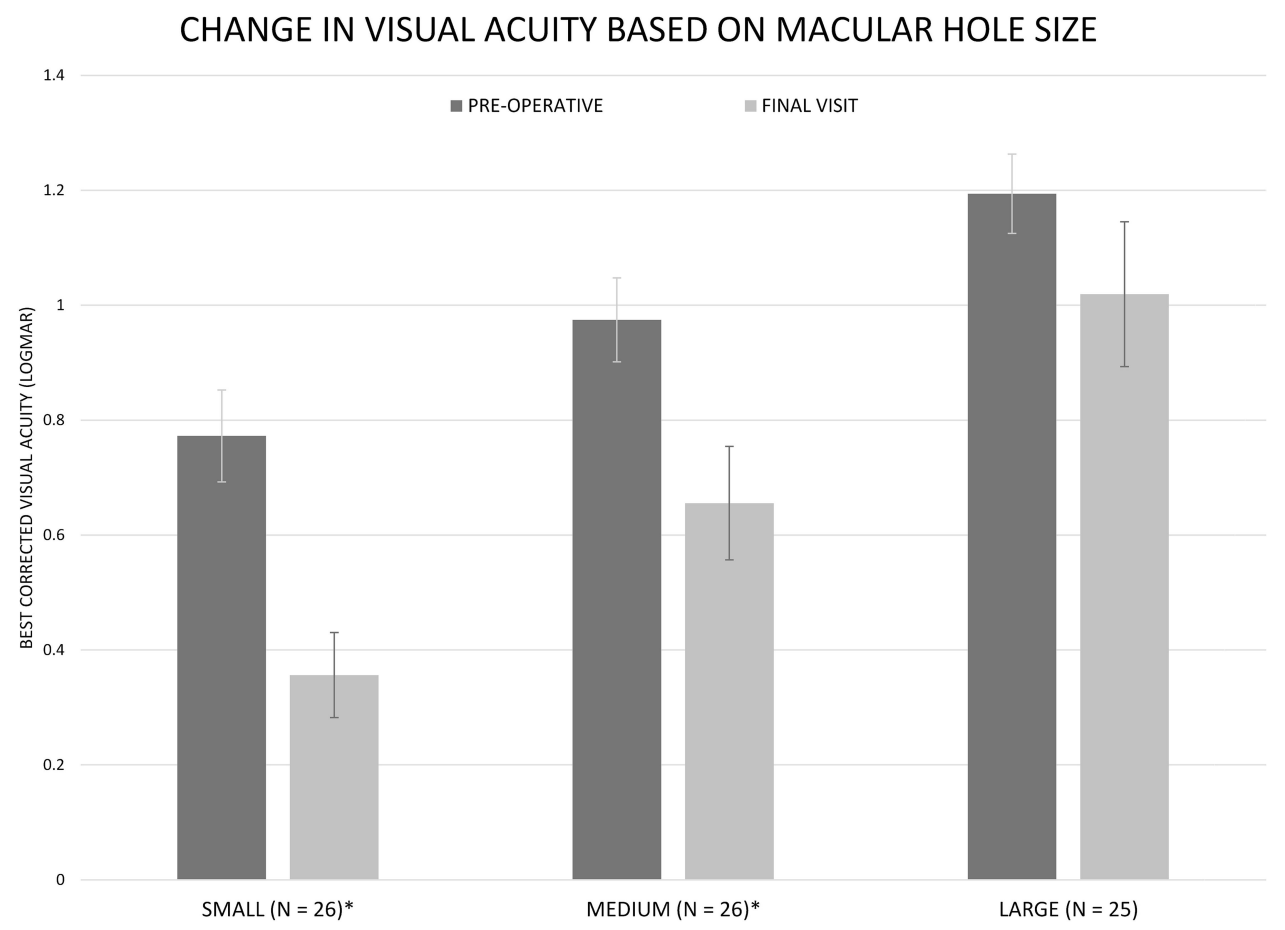

Figure 5 The mean best-corrected visual acuity pre-operatively and at final visit based on size of macular hole on optical coherence tomography (small <250 microns, medium 250 to 400 microns, large $>400$ microns). Error bars represent standard error of means and $(*)$ indicates statistically significant data ( $<<0.05)$.

ILM peel; ${ }^{19,23,24}$ however, all closure rates have largely been dependent on duration of symptoms, stage, and size. $^{25-28}$ Even though our SSC rate was on the lower end of previously reported rates our cohort includes a high percentage of stage $4 \mathrm{MHs}(44.8 \%)$, large MHs (32.4\%), and eyes with pathologic myopia (13.0\%). All of these characteristics portend lower closure rates. ${ }^{25,26,29,30}$ When evaluating eyes without pathologic myopia, stage 2 or $3 \mathrm{MHs}$ had a much higher SSC rate at $92.3 \%$. Similarly, small or medium MHs in eyes without pathologic myopia had a higher SSC rate at $88.6 \%$. Our SSC rate was $100 \%$ for stage $2(n=20)$ and small $(n=21)$ MHs. Interestingly, in our cohort of patients, the SSC rate is almost identical between the acute and chronic MHs (82.9\% vs $87.5 \%)$. Given the small sample size in the chronic MH cohort $(n=16)$, especially in comparison to the more robust acute MH cohort $(n=41)$, it is difficult to draw significant conclusions from these findings. Importantly, successful closure at the final visit occurred in all of the acute MHs while no more chronic MHs were successfully closed with further intervention. The recalcitrant nature of the chronic MHs in our cohort is consistent with the observations from previous studies that these pose a treatment challenge. ${ }^{31}$ We did not attempt closure with ILM flap inversion or amniotic membrane transplant. ${ }^{32,33}$
There has been concern raised previously that cataract surgery and, specifically, the development of CME following cataract surgery are risk factors in recurrent $\mathrm{MHs} .{ }^{34-36}$ Within the recurrent MHs in the presented study group, 1 $(3.6 \%)$ eye recently underwent cataract surgery and subsequently developed CME. In total, 8 (61.5\%) eyes had $\mathrm{CME}$ at the time of recurrence with 4 pseudophakic and 4 phakic eyes. Epiretinal membrane, uveitis, and exudative macular degeneration accounted for the majority of the etiologies of CME. The eyes with uveitis and exudative macular degeneration were actively being treated with intraocular injections and still developed significant CME resulting in recurrent MHs. These findings support the concern that $\mathrm{CME}$ is a risk factor for recurrence in eyes that have undergone $\mathrm{MH}$ repair and should be aggressively managed.

Pathologic myopia is another condition that poses a challenge to $\mathrm{MH}$ closure with previously reported closure rates with ILM peel between 60 and $100 \%$. $^{30,37,38}$ In our study, we had a final closure rate of $72.7 \%$ in eyes with pathologic myopia; however, only a $45.4 \%$ SSC rate. Evaluating the pathologic myopia eyes against all other eyes reveals a significantly better SSC rate in those without pathologic myopia ( $p=0.011$ ). Only two stage 2 and two small MHs recurred in our cohort and they all had pathologic myopia. One small, stage $2 \mathrm{MH}$ that recurred 


\section{A SINGLE SURGERY CLOSURE RATE (EXCLUDING EYES WITH}

PATHOLOGIC MYOPIA)

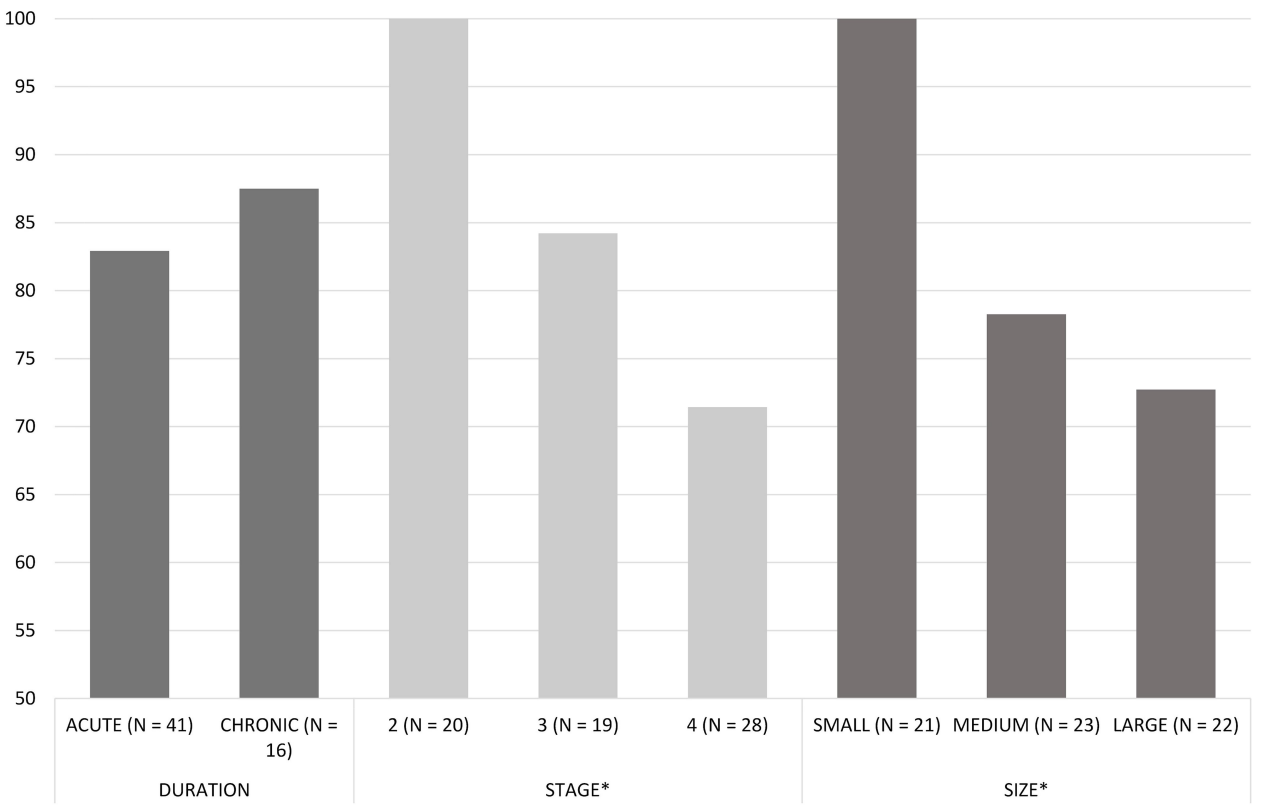

\section{B FINAL CLOSURE RATE (EXCLUDING EYES WITH PATHOLOGIC MYOPIA)}

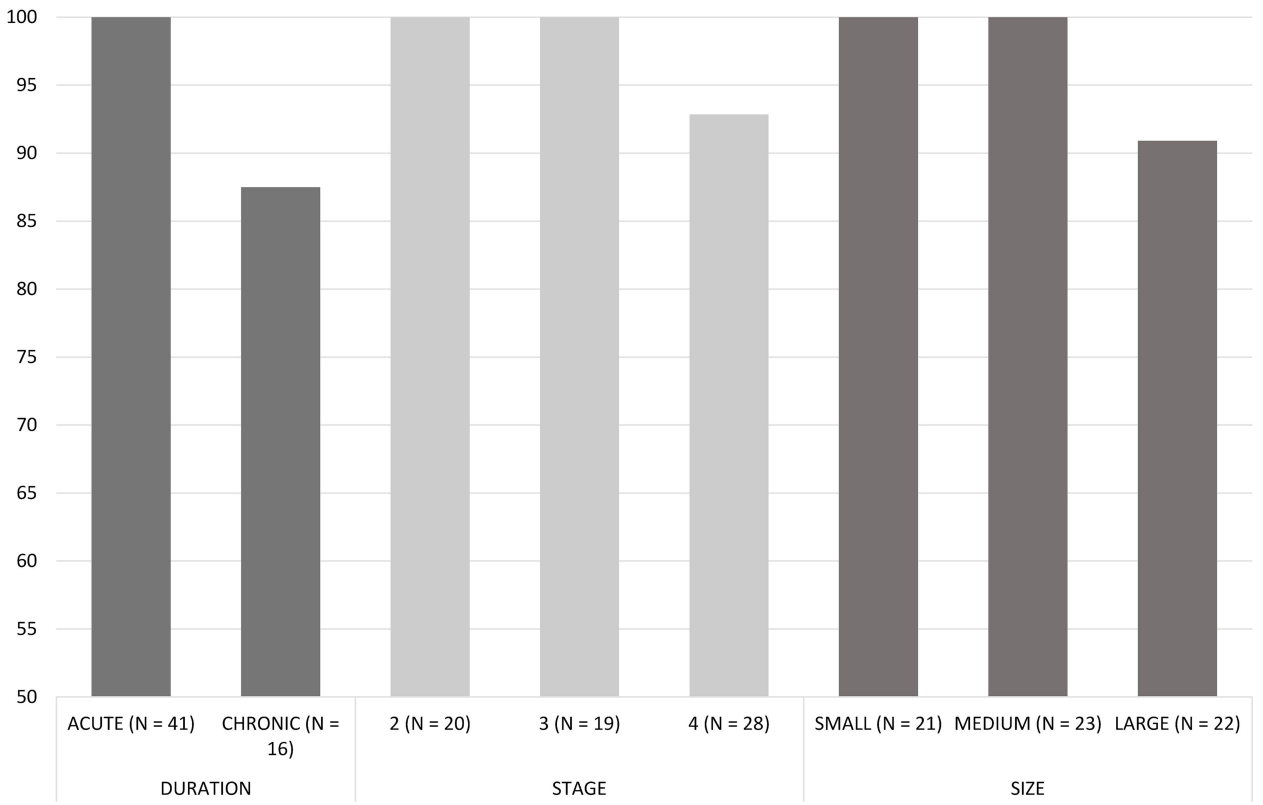

Figure 6 In eyes without pathologic myopia, (A) single surgery closure rates and (B) final closure rates stratified by duration of symptoms, stage of macular hole and size of macular hole. The single surgery closure was defined as successful macular hole closure after one intervention while final closure was defined by macular hole closure at the final follow-up visit independent of number of interventions. $\left({ }^{*}\right)$ indicates statistically significant data $(p<0.05)$.

shortly after initial surgery was also associated with foveoschisis which poses further challenge. Ikuno et al reported on 11 eyes with MHs associated with myopic foveoschisis and noted closure in only 4 eyes. ${ }^{39}$ These poorer outcomes with pathologic myopia and foveoschisis have resulted in research into alternative treatment options such as an inverted ILM flap and macular buckling. ${ }^{40,41}$ Neither of these were utilized in this study cohort. 
Table 3 Complications

\begin{tabular}{|c|c|c|}
\hline $\begin{array}{l}\text { Pre-Operative IOP } \\
\text { Mean } \pm \text { S.E.M. }\end{array}$ & $16.3 \pm 0.3$ & P-value* \\
\hline $\begin{array}{l}\text { Final Visit IOP } \\
\text { Mean } \pm \text { S.E.M. }\end{array}$ & $15.5 \pm 0.2$ & $<0.05$ \\
\hline $\begin{array}{l}\text { IOP }>25 \mathrm{mmHg} \text { within } 2 \text { Months } \\
\text { Eyes (\%) }\end{array}$ & $18(23.1 \%)$ & \\
\hline $\begin{array}{l}\text { Endophthalmitis } \\
\text { Eyes (\%) }\end{array}$ & $0(0 \%)$ & \\
\hline $\begin{array}{l}\text { Retinal Detachment } \\
\text { Eyes (\%) }\end{array}$ & I (I.3\%) & \\
\hline $\begin{array}{l}\text { Ahmed Valve Placement }{ }^{\dagger} \\
\text { Eyes (\%) }\end{array}$ & $2(2.6 \%)$ & \\
\hline $\begin{array}{l}\text { Cataract Progression/Surgery } \\
\text { Eyes (\%) }\end{array}$ & $52(96.3 \%)^{\ddagger}$ & \\
\hline $\begin{array}{l}\text { Cataract Surgery } \\
\text { Eyes }(\%)\end{array}$ & $35(64.8 \%)^{\ddagger}$ & \\
\hline $\begin{array}{l}\text { Recurrent Macular Hole } \\
\text { Eyes (\%) }\end{array}$ & $13(16.7 \%)$ & \\
\hline \multicolumn{3}{|c|}{ Recurrent Macular Hole Associated Findings } \\
\hline $\begin{array}{l}\text { Lens Status Prior to Initial Surgery } \\
\text { Pseudophakic: Phakic }\end{array}$ & 5: 8 & \\
\hline $\begin{array}{l}\text { High Myopia } \\
\text { Eyes (\%) }\end{array}$ & $6(46.2 \%)$ & \\
\hline $\begin{array}{l}\text { Uveitis } \\
\text { Eyes (\%) }\end{array}$ & I (7.7\%) & \\
\hline $\begin{array}{l}\text { Cystoid Macular Edema** } \\
\text { Eyes (\%) }\end{array}$ & $8(61.5 \%)$ & \\
\hline $\begin{array}{l}\text { Cataract Surgery Prior to Recurrence } \\
\text { Eyes (\% of phakic eyes) }\end{array}$ & I (I2.5\%) & \\
\hline $\begin{array}{l}\text { Cataract Surgery After Recurrence }{ }^{\dagger \dagger} \\
\text { Eyes (\% of phakic eyes) }\end{array}$ & $4(50.0 \%)$ & \\
\hline $\begin{array}{l}\text { Time to Recurrence (Days) } \\
\text { Mean } \pm \text { S.E.M. }\end{array}$ & $223.0 \pm 87.6$ & \\
\hline
\end{tabular}

Notes: *Compared to pre-operative value. ${ }^{\dagger}$ Both eyes had pre-existing glaucoma with intolerance to drop therapy. ${ }^{\ddagger}$ Based on phakic eyes (54) at pre-operative assessment. **Etiology of CME: epiretinal membrane 3 eyes, exudative macular

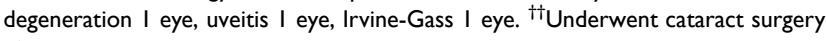
after initial recurrence and re-closure without complication.

Abbreviations: IOP, intraocular pressure; S.E.M., standard error of means.

Repair of MH with TA-assisted ILM peel has routinely demonstrated BCVA improvements. ${ }^{19,22-24,42}$ The eyes in the presented study were noted to have a significant improvement in BCVA at their final visit along with a significant improvement noted at each follow-up after 2 months. Continued improvement was appreciated in BCVA from the 1- to 2-year follow-up visit, which is consistent with previous reports of BCVA improvement even more than 1 year removed from $\mathrm{MH}$ repair. ${ }^{43}$ Sixteen eyes had 4-years of follow-up achieving a final mean BCVA of $0.32 \pm 0.04(20 / 42) \log$ MAR with 10 eyes having a final BCVA of at least 20/30. This demonstrates the long-term efficacy of this treatment strategy with significant BCVA benefits that continue to show improvement well after surgery. As was discussed above, these BCVA results are in a cohort of eyes with a high percentage of stage 4 or large MHs. Overall, the MH closure rates (especially at final visit) and BCVA improvements noted with TA-assisted ILM peel in our cohort are comparable to prior studies. ${ }^{19-21}$ Treatment of MHs associated with pathologic myopia presented a challenge to successful closure in our cohort as did the development of postoperative CME.

\section{Vital Dye Selection}

As previously discussed, while ILM peeling has become a commonplace practice in the treatment of MHs the choice of vital dye for visualization is controversial. Many studies have evaluated the various dyes to determine which offer the best results. Wang et al performed a metaanalysis of these studies to better elucidate the top performing vital dye and determined that there was no statistical difference in the closure rates between TA, BBG, ICG, and TB. TA and BBG, however, performed the best in regards to BCVA response. ${ }^{2} \mathrm{Li}$ et al similarly performed a meta-analysis of these studies which once again found minimal statistical differences in results with these dyes, but most notable was the improved BCVA results with TB and BBG compared to others. ${ }^{44}$ Much concern has been raised regarding possible retinal toxicity from the use of ICG which has been theorized as the culprit for the poorer visual response with this dye along with reported visual field defects. ${ }^{4}$ Although there is evidence that lower concentrations of ICG may be less toxic to the retina when provided in abbreviated doses. ${ }^{5}$ Trypan blue is similar to TA as it does not truly stain the ILM - as ICG and BBG do -and there has been concerns about possible retinal toxicity associated with TB especially with longer exposure. ${ }^{5,45,46}$ Finally, BBG has been met with the most optimism and appears to be the least toxic dye though cases of toxicity have been reported. ${ }^{8,13,14,47}$ In addition, there have been previous reports of fungal endophthalmitis associated with the compounding process of $\mathrm{BBG},{ }^{48}$ 
although the recent food and drug administration approved BBG (TissueBlue: Dutch Ophthalmic USA; Exeter, NH) may obviate this. Within the United States ICG remains the preferred vital dye for ILM peeling, but BBG has been gaining popularity and is the preferred dye by international retina surgeons. In the most recent PAT survey, the percentages of stains used by US retina surgeons were as follows: ICG $-75 \%, \mathrm{BBG}-16 \%$, TA $-5 \%$, and $\mathrm{TB}-1 \%{ }^{12}$

Although there was previously evidence of retinal toxicity noted with the use of TA, these were likely due to the preservatives found in older formulations of TA and none have been reported with MH repair. ${ }^{11,21,49}$ The advent of preservative-free TA, which is now available and used in this study, appears to be safe. In support of this, even subfoveal TA which can infrequently occur during $\mathrm{MH}$ repair does not cause toxicity with TA crystals resolving over weeks to months. ${ }^{50}$ Within our large cohort of eyes, neither signs of retinal toxicity nor retained TA were noted throughout the course of the study. Triamcinolone acetonide has been used in the posterior segment for decades in other applications - such as the treatment of macular edema $^{10}$ and for staining of vitreous ${ }^{9}$ - with a strong safety profile. Its commonplace use in staining of vitreous means that it is readily available for surgical use and can provide both functions intraoperatively rather than using separate dyes. In addition, we feel that the anti-inflammatory properties of TA offer a theoretical benefit to macular edema that is not offered by other vital dyes and find the optimal contrast provided by the white crystals preferrable to the other dyes.

\section{Predictors of Outcomes}

Multiple MH grading schemas have previously been proposed to assist in the preoperative assessment. The proposed metric by Gass, prior to the widespread use of OCT images, utilized slit-lamp biomicroscopic findings to stage MHs on a 1 to 4 scale largely predicated on degree of vitreous attachment or detachment to the macula. ${ }^{15}$ More recently, the International Vitreomacular Traction Study (IVTS) released a revised grading schema utilizing OCT findings. This schema is based upon the diameter of the $\mathrm{MH}$ at its smallest width and broken down into small $(\leq 250 \mu \mathrm{m})$, medium $(250$ to $400 \mu \mathrm{m})$, and large $(\geq 400$ $\mu \mathrm{m}) .{ }^{16}$ It remains unclear whether either of these metrics or even duration of $\mathrm{MH}$ provides a better predictor of $\mathrm{MH}$ closure rates and BCVA outcomes.
As discussed above, previous studies have been mixed regarding the impact of Gass staging, IVTS size, and symptom duration on closure rate and BCVA. ${ }^{25-28}$ Within our study cohort, stage 2 and 3 MHs and small MHs appeared to be associated with the best BCVA and closure results. While duration did not appear to be predictive of outcomes there were some signs that acute MHs were more responsive to therapy. First, while having nearly identical SSC rates all of the acute MHs were successfully closed with subsequent interventions, none of the chronic MHs responded. Secondly, the BCVA for acute MHs significantly improved from pre-operative to final visit, but the chronic $\mathrm{MH}$ improvement was not significant. Despite this, the difference in BCVA improvement between the acute and chronic MH cohorts was not significant. The stage, size, and, to a lesser degree, duration all offer information pre-operatively that are important predictors of successful MH treatment and should routinely be noted when evaluating such patients.

\section{Intraocular Gas and Face-Down Posturing}

Various intraocular gases and face-down posturing protocols have been proposed for MH repair and, similar to vital dye selection, these choices remain controversial. The most commonly utilized intraocular gases in $\mathrm{MH}$ repair are C3F8 and sulfur hexafluoride (SF6). These gases most significantly differ by their longevity in the eye with studies demonstrating approximately 18.0 days for SF6 and 67.7 days for $\mathrm{C} 3 \mathrm{~F} 8 .^{51}$ Based on this longevity, SF6 has the benefit of more rapid visual recovery while $\mathrm{C} 3 \mathrm{~F} 8$ a longer length of tamponade of the $\mathrm{MH}$. Comparative studies have shown nonsignificant differences in closure rates between these gases, ${ }^{52}$ although there are reports of better visual outcomes with the use of $\mathrm{C} 3 \mathrm{~F} 8 .{ }^{53}$ Similarly, there have been numerous proposed face-down posturing regimens ranging from those without any such positioning to those with 2 weeks of positioning. ${ }^{26,54}$ Over the past decade, there has been a shift towards shorter face-down posturing regimens with the most recent PAT survey reporting $5 \%$ of US retina surgeons posturing for 8-14 days, $46 \%$ for $5-7$ days, and $48 \%$ for less than 5 days. ${ }^{12}$ A Cochrane review reported the benefit of face down posturing in large MHs but did not note a definitive benefit to smaller MHs. ${ }^{55}$ It has been our practice to utilize $\mathrm{C} 3 \mathrm{~F} 8$ and 1-2 weeks of face-down posturing for all MH repairs. We feel that the longer gas fill and tamponade provided by C3F8 is key to the successful closure of MHs and the benefit of quicker visual recovery with SF6 is not 
outweighed by this point. Our face-down posturing relies on a longer regimen for more complicated cases that have a higher likelihood of reopening. Despite the high percentage of stage $4 \mathrm{MHs}$, large MHs and those with pathologic myopia, we feel the high closure rate can partially be attributed to the gas selection and face-down posturing regimen.

\section{Complications}

The complication rates in this study continue to support the previous safety profile of TA-assisted ILM peel. ${ }^{21}$ While there has been a theoretical concern for endophthalmitis from intraocular steroid use during MH surgery it has not been supported by the data and we did not have any examples of endophthalmitis in our cohort. ${ }^{56}$ Our rate of cataract progression or surgery in phakic patients is quite high at $96.3 \%$, but this is a common complication of pars plana vitrectomy and gas tamponade without intraocular steroid application. Elhusseiny et al reported a rate of cataract surgery of $91.2 \%$ in eyes following $\mathrm{MH}$ repair with ICG with long-term follow-up. ${ }^{20}$ Another theoretical concern has been the development of steroid-induced glaucoma. This cohort of eyes had 2 cases that required Ahmed valve placement for management of glaucoma within the first 3 months after MH surgery. Both of these eyes were on maximal tolerated therapy for primary openangle glaucoma prior to $\mathrm{MH}$ surgery with persistently uncontrollable IOP after $\mathrm{MH}$ surgery. We previously reported a similar rate of glaucoma in eyes treated with TA-assisted ILM peel for epiretinal membranes. ${ }^{57}$ In both cases, it is unclear whether this progression can be directly correlated with the brief intraoperative exposure to TA as there is an inherent risk of elevated IOP associated with vitrectomy alone. ${ }^{58,59}$ This risk is further increased with the use of C3F8 gas tamponade. ${ }^{58-60}$ Overall, we noted an IOP greater than $25 \mathrm{mmHg}$ in $23.1 \%$ of eyes within the first 2 months after surgery. This rate is consistent with previously published data in eyes undergoing vitrectomy and $\mathrm{C} 3 \mathrm{~F} 8$ gas tamponade, $20-40 \%{ }^{61-63}$

\section{Limitations}

The main limitations of this study are in the design both being retrospective and lacking a control or comparative arm. Not having an alternative dye for comparison makes it difficult to make broad statements regarding the BCVA and closure rate results in our group. In addition, having all the surgeries performed by a single surgeon limits the broader applications as varying surgeon skill levels may find different results than those presented. Also, more recent research has proposed the use of amniotic membranes or inverting the ILM within the $\mathrm{MH}$ to assist in larger $\mathrm{MHs}$ and those associated with pathologic myopia which was not available in the treatment of any of these eyes. ${ }^{32,33,64}$ Such novel approaches may have further improved the results in this cohort of eyes. Finally, being a tertiary referral center, complex MHs are often referred from other ophthalmologists and, occasionally, other retina surgeons. We elected to include all such cases in the study cohort.

\section{Conclusion}

Triamcinolone acetonide-assisted ILM peel offers a safe treatment for $\mathrm{MH}$ with comparable visual acuity results and closure rates to prior reports. These results are consistent after many years of follow-up. When evaluating patients pre-operatively, it is imperative to note the stage, size, and duration of the $\mathrm{MH}$ for proper patient education regarding expected outcomes. Eyes with pre-existing glaucoma may experience progression post-operatively which may be due to the intraoperative steroid exposure or the surgical procedure and gas tamponade. Pathologic myopia offers a challenge to successful macular hole closure and patients may benefit from alternative surgical approaches. Post-operative macular edema is a significant risk for recurrence and should be managed aggressively.

\section{Disclosure}

The authors report no conflicts of interest in this work.

\section{References}

1. Spiteri Cornish K, Lois N, Scott NW, et al. Vitrectomy with internal limiting membrane peeling versus no peeling for idiopathic full-thickness macular hole. Ophthalmology. 2014;121(3):649-655. doi:10.1016/j.ophtha.2013.10.020

2. Wang XW, Long Y, Gu YS, Guo DY. Outcomes of 4 surgical adjuvants used for internal limiting membrane peeling in macular hole surgery: a systematic review and network meta-analysis. Int J Ophthalmol. 2020;13(3):481-487. doi:10.18240/ijo.2020.03.17

3. Stanescu-Segall D, Jackson TL. Vital staining with indocyanine green: a review of the clinical and experimental studies relating to safety. Eye (Lond). 2009;23(3):504-518. doi:10.1038/eye.2008.249

4. Wu Y, Zhu W, Xu D, et al. Indocyanine green-assisted internal limiting membrane peeling in macular hole surgery: a meta-analysis. PLoS One. 2012;7(11):e48405. doi:10.1371/journal.pone.0048405

5. Kodjikian L, Richter T, Halberstadt M, et al. Toxic effects of indocyanine green, infracyanine green, and trypan blue on the human retinal pigmented epithelium. Graefes Arch Clin Exp Ophthalmol. 2005;243 (9):917-925. doi:10.1007/s00417-004-1121-6

6. Gass CA, Haritoglou C, Schaumberger M, Kampik A. Functional outcome of macular hole surgery with and without indocyanine green-assisted peeling of the internal limiting membrane. Graefes Arch Clin Exp Ophthalmol. 2003;241(9):716-720. doi:10.1007/ s00417-003-0710-0 
7. Enaida H, Hisatomi T, Hata Y, et al. Brilliant blue G selectively stains the internal limiting membrane/brilliant blue G-assisted membrane peeling. Retina. 2006;26(6):631-636. doi:10.1097/01.iae.0000236469.71443.aa

8. Jindal A, Pathengay A, Mithal K, Chhablani J, Pappuru RR, Flynn HW. Macular toxicity following brilliant blue G-assisted macular hole surgery - a report of three cases. Nepal J Ophthalmol. 2014;6 (11):98-101. doi:10.3126/nepjoph.v6i1.10779

9. Sakamoto T, Miyazaki M, Hisatomi T, et al. Triamcinolone-assisted pars plana vitrectomy improves the surgical procedures and decreases the postoperative blood-ocular barrier breakdown. Graefes Arch Clin Exp Ophthalmol. 2002;240(6):423-429. doi:10.1007/s00417-0020454-2

10. Thorne JE, Sugar EA, Holbrook JT, et al. Periocular triamcinolone vs. intravitreal triamcinolone vs. intravitreal dexamethasone implant for the treatment of uveitic macular edema: the PeriOcular vs. INTravitreal corticosteroids for uveitic macular edema (POINT) Trial. Ophthalmology. 2019;126(2):283-295. doi:10.1016/j. ophtha.2018.08.021

11. Macky TA, Helmy D, El Shazly N. Retinal toxicity of triamcinolone's vehicle (benzyl alcohol): an electrophysiologic and electron microscopic study. Graefes Arch Clin Exp Ophthalmol. 2007;245 (6):817-824. doi:10.1007/s00417-006-0459-3

12. Hahn P. ASRS 2020 Preferences and Trends Membership Survey. Chicago, IL: American Society of Retina Specialists; 2020.

13. Almeida FPP, De Lucca AC, Scott IU, Jorge R, Messias A. Accidental subretinal brilliant blue $\mathrm{G}$ migration during internal limiting membrane peeling surgery. JAMA Ophthalmol. 2015;133 (1):85-88. doi:10.1001/jamaophthalmol.2014.3869

14. Venkatesh R, Aseem A, Jain K, Yadav NK. Combined brilliant blue $\mathrm{G}$ and xenon light induced outer retinal layer damage following macular hole surgery. Indian J Ophthalmol. 2020;68(1):247-249. doi:10.4103/ijo.IJO_1386_19

15. Gass JD. Reappraisal of biomicroscopic classification of stages of development of a macular hole. Am J Ophthalmol. 1995;119 (6):752-759. doi:10.1016/s0002-9394(14)72781-3

16. Duker JS, Kaiser PK, Binder S, et al. The International Vitreomacular Traction Study Group classification of vitreomacular adhesion, traction, and macular hole. Ophthalmology. 2013;120(12):2611-2619. doi:10.1016/j.ophtha.2013.07.042

17. Kumar A, Chawla R, Kumawat D, Pillay G. Insight into high myopia and the macula. Indian $J$ Ophthalmol. 2017;65(2):85-91. doi:10.4103/ijo.IJO_863_16

18. Faghihi H, Hajizadeh F, Riazi-Esfahani M. Optical coherence tomographic findings in highly myopic eyes. J Ophthalmic Vis Res. 2010;5 (2):110-121

19. Kumar A, Gogia V, Shah VM, Nag TC. Comparative evaluation of anatomical and functional outcomes using brilliant blue $G$ versus triamcinolone assisted ILM peeling in macular hole surgery in Indian population. Graefes Arch Clin Exp Ophthalmol. 2011;249 (7):987-995. doi:10.1007/s00417-010-1609-1

20. Elhusseiny AM, Schwartz SG, Flynn HW Jr, Smiddy WE. Long-term outcomes after macular hole surgery. Ophthalmol Retina. 2020;4 (4):369-376. doi:10.1016/j.oret.2019.09.015

21. Kampougeris G, Cheema R, McPherson R, Gorman C. Safety of Triamcinolone acetonide (TA)-assisted pars plana vitrectomy in macular hole surgery. Eye (Lond). 2007;21(5):591-594. doi:10.1038/sj.eye. 6702265

22. Azuma K, Noda Y, Hirasawa K, Ueta T. Brilliant blue G-assisted internal limiting membrane peeling for macular hole: a systematic review of literature and meta-analysis. Retina. 2016;36(5):851-858. doi:10.1097/iae.0000000000000968

23. Mochizuki N, Yamamoto T, Enaida H, Ishibashi T, Yamashita H. Long-term outcomes of 3 surgical adjuvants used for internal limiting membrane peeling in idiopathic macular hole surgery. Jpn J Ophthalmol. 2014;58(6):455-461. doi:10.1007/s10384-014-0345-1
24. Nomoto H, Shiraga F, Yamaji H, et al. Macular hole surgery with triamcinolone acetonide-assisted internal limiting membrane peeling: one-year results. Retina. 2008;28(3):427-432. doi:10.1097/ IAE.0b013e31815ec2f1

25. Williamson $\mathrm{TH}$, Lee E. Idiopathic macular hole: analysis of visual outcomes and the use of indocyanine green or brilliant blue for internal limiting membrane peel. Graefes Arch Clin Exp Ophthalmol. 2014;252(3):395-400. doi:10.1007/s00417-0132477-2

26. Ip MS, Baker BJ, Duker JS, et al. Anatomical outcomes of surgery for idiopathic macular hole as determined by optical coherence tomography. Arch Ophthalmol. 2002;120(1):29-35. doi:10.1001/ archopht.120.1.29

27. Kang HK, Chang AA, Beaumont PE. The macular hole: report of an Australian surgical series and meta-analysis of the literature. Clin Exp Ophthalmol. 2000;28(4):298-308. doi:10.1046/j.14429071.2000.00329.x

28. Jaycock PD, Bunce C, Xing W, et al. Outcomes of macular hole surgery: implications for surgical management and clinical governance. Eye (Lond). 2005;19(8):879-884. doi:10.1038/sj. eye. 6701679

29. Kumar A, Prakash G, Singh RP. Indocyanine green enhanced maculorhexis in macular hole surgery. Indian J Ophthalmol. 2002;50 (2):123-126.

30. Patel SC, Loo RH, Thompson JT, Sjaarda RN. Macular hole surgery in high myopia. Ophthalmology. 2001;108(2):377-380. doi:10.1016/ S0161-6420(00)00532-7

31. Yu Y, Liang X, Wang Z, et al. Internal limiting membrane peeling and air tamponade for stage III and stage IV idiopathic macular hole. Retina. 2020;40(1):66-74. doi:10.1097/iae.0000000000002340

32. Chen G, Tzekov R, Jiang F, Mao S, Tong Y, Li W. Inverted ILM flap technique versus conventional ILM peeling for idiopathic large macular holes: a meta-analysis of randomized controlled trials. PLoS One. 2020;15(7):e0236431. doi:10.1371/journal.pone.0236431

33. Caporossi T, Pacini B, De Angelis L, Rizzo S. Amniotic membrane plug to promote chronicpost-traumatic macular hole closure. Ophthalmic Surg Lasers Imaging Retina. 2019;51(1):50-52. doi:10.3928/23258160-20191211-07

34. Bhatnagar P, Kaiser PK, Smith SD, Meisler DM, Lewis H, Sears JE. Reopening of previously closed macular holes after cataract extraction. Am J Ophthalmol. 2007;144(2):252-259. doi:10.1016/j. ajo.2007.04.041

35. Paques M, Massin P, Blain P, Duquesnoy AS, Gaudric A. Long-term incidence of reopening of macular holes. Ophthalmology. 2000;107 (4):760-5; discussion 766. doi:10.1016/s0161-6420(99)00182-7

36. Abbey AM, Van Laere L, Shah AR, Hassan TS. Recurrent macular holes in the era of small-gauge vitrectomy: a review of incidence, risk factors, and outcomes. Retina. 2017;37(5):921-924. doi:10.1097/ iae.0000000000001252

37. Wu -T-T, Kung Y-H. Comparison of anatomical and visual outcomes of macular hole surgery in patients with high myopia vs. non-high myopia: a case-control study using optical coherence tomography. Graefes Arch Clin Exp Ophthalmol. 2012;250(3):327-331. doi:10.1007/s00417-011-1821-7

38. Qu J, Zhao M, Jiang Y, Li X. Vitrectomy outcomes in eyes with high myopic macular hole without retinal detachment. Retina. 2012;32 (2):275-280. doi:10.1097/IAE.0b013e31821a8901

39. Ikuno Y, Sayanagi K, Soga K, Oshima Y, Ohji M, Tano Y. Foveal anatomical status and surgical results in vitrectomy for myopic foveoschisis. Jpn J Ophthalmol. 2008;52(4):269-276. doi:10.1007/ s10384-008-0544-8

40. Michalewska Z, Michalewski J, Dulczewska-Cichecka K, Nawrocki J. Inverted internal limiting membrane flap technique for surgical repair of myopic macular holes. Retina. 2014;34(4):664-669. doi:10.1097/iae.0000000000000042 
41. Cacciamani A, Lazzeri S, Rossi T, et al. Adjustable macular buckling for full-thickness macular hole with Foveoschisis in highly myopic eyes: long-term anatomical and functional results. Retina. 2016;36 (4):709-716. doi:10.1097/iae.0000000000000802

42. Tewari A, Almony A, Shah GK. Macular hole closure with triamcinolone-assisted internal limiting membrane peeling. Retina. 2008;28(9):1276-1279. doi:10.1097/IAE.0b013e31817d8be1

43. Leonard RE, Smiddy WE, Flynn HW, Feuer W. Long-term visual outcomes in patients with successful macular hole surgery. Ophthalmology. 1997;104(10):1648-1652. doi:10.1016/S0161-6420(97)30071-2

44. Li SS, You R, Li M, et al. Internal limiting membrane peeling with different dyes in the surgery of idiopathic macular hole: a systematic review of literature and network meta-analysis. Int $J$ Ophthalmol. 2019;12(12):1917-1928. doi:10.18240/ijo.2019.12.15

45. Rishi P, Rishi E, Kothari A. Retinal pigment epithelial changes after trypan blue-assisted internal limiting membrane peeling for idiopathic macular hole. Oman $J$ Ophthalmol. 2011;4(1):37-38. doi:10.4103/0974-620x.77663

46. Jain S, Kishore K, Sharma YR. Progressive atrophy of retinal pigment epithelium after trypan-blue-assisted ILM peeling for macular hole surgery. Indian $J$ Ophthalmol. 2013;61(5):235-237. doi:10.4103/0301-4738.111180

47. Balaiya S, Brar VS, Murthy RK, Chalam KV. Comparative in vitro safety analysis of dyes for chromovitrectomy: indocyanine green, brilliant blue green, bromophenol blue, and infracyanine green. Retina. 2011;31(6):1128-1136. doi:10.1097/IAE.0b013e3181fe543a

48. Mikosz CA, Smith RM, Kim M, et al. Fungal endophthalmitis associated with compounded products. Emerg Infect Dis. 2014;20 (2):248-256. doi:10.3201/eid2002.131257

49. Scupola A, Mastrocola A, Sasso P, et al. Assessment of retinal function before and after idiopathic macular hole surgery. Am J Ophthalmol. 2013;156(1):132-139.e1. doi:10.1016/j.ajo.2013.02.007

50. Payne JF, Bergstrom C, Yan J, Aaberg TM Sr, Srivastava SK. Residual triamcinolone acetonide sequestered in the fovea after macular hole repair. Retina. 2011;31(1):148-153. doi:10.1097/ IAE.0b013e3181ea48a1

51. Kontos A, Tee J, Stuart A, Shalchi Z, Williamson TH. Duration of intraocular gases following vitreoretinal surgery. Graefes Arch Clin Exp Ophthalmol. 2017;255(2):231-236. doi:10.1007/s00417-016-3438-3

52. Hecht I, Mimouni M, Blumenthal EZ, Barak Y. Sulfur Hexafluoride (SF (6)) versus Perfluoropropane $(\mathrm{C}(3) \mathrm{F}(8))$ in the intraoperative management of macular holes: a systematic review and meta-analysis. J Ophthalmol. 2019;2019:1820850. doi:10.1155/2019/1820850

53. Zarranz-Ventura J, Sallam A, Keane P, Sim D, Kirkpatrick N, Johnston R. Macular hole surgery: does C3F8 provide better anatomical and functional outcomes than SF6 and $\mathrm{C} 2 \mathrm{~F} 6$ ? Invest Ophthalmol Vis Sci. 2013;54(15):5777.
54. Guerrero-Naranjo JL, Cortés-Luna C, Morales-Cantón V, et al. Cirugía de agujero macular sin posición «boca abajo» postoperatoria. Estudio piloto. [Macular hole surgery without postoperatory «face» down positioning. Pilot study]. Arch Soc Esp Oftalmol. 2006;81(6):321-326. doi:10.4321/s0365-66912006000600005

55. Solebo AL, Lange CA, Bunce C, Bainbridge JW. Face-down positioning or posturing after macular hole surgery. Cochrane Database Syst Rev. 2011;12:Cd008228. doi:10.1002/14651858.CD008228.pub2

56. Sakamoto T, Enaida H, Kubota $T$, et al. Incidence of acute endophthalmitis after triamcinolone-assisted pars plana vitrectomy. Am J Ophthalmol. 2004;138(1):137-138. doi:10.1016/j. ajo.2004.02.072

57. Asahi MG, Wallsh JO, Gallemore RP. Outcomes of epiretinal membrane removal utilizing triamcinolone acetonide visualization and internal limiting membrane forceps. Clin Ophthalmol. 2020;14:3913-3921. doi:10.2147/opth.s279611

58. Chen CJ. Glaucoma after macular hole surgery. Ophthalmology. 1998;105(1):94-9; discussion 99-100. doi:10.1016/s0161-6420(98) 91470-1

59. Hakimbashi M, Rezaei K. Rate of elevated intraocular pressure post primary vitrectomy surgery. Invest Ophthalmol Vis Sci. 2013;54 (15):5802.

60. Fang Y, Long Q, Wang X, Jiang R, Sun X. Intraocular pressure 1 year after vitrectomy in eyes without a history of glaucoma or ocular hypertension. Clin Ophthalmol. 2017;11:2091-2097. doi:10.2147/ OPTH.S144985

61. Briand S, Chalifoux E, Tourville E, et al. Prospective randomized trial: outcomes of $\mathrm{SF}_{6}$ versus $\mathrm{C}_{3} \mathrm{~F}_{8}$ in macular hole surgery. Can J Ophthalmol. 2015;50(2):95-100. doi:10.1016/j.jcjo.2014.12.006

62. Chen PP, Thompson JT. Risk factors for elevated intraocular pressure after the use of intraocular gases in vitreoretinal surgery. Ophthalmic Surg Lasers. 1997;28(1):37-42.

63. Mittra RA, Pollack JS, Dev S, et al. The use of topical aqueous suppressants in the prevention of postoperative intraocular pressure elevation after pars plana vitrectomy with long-acting gas tamponade. Ophthalmology. 2000;107(3):588-592. doi:10.1016/s0161-6420(99) 00083-4

64. Huang YH, Tsai DC, Wang LC, Chen SJ. Comparison between cryopreserved and dehydrated human amniotic membrane graft in treating challenging cases with macular hole and macular hole retinal detachment. J Ophthalmol. 2020;2020:9157518. doi:10.1155/2020/ 9157518
Clinical Ophthalmology

\section{Publish your work in this journal}

Clinical Ophthalmology is an international, peer-reviewed journal covering all subspecialties within ophthalmology. Key topics include: Optometry; Visual science; Pharmacology and drug therapy in eye diseases; Basic Sciences; Primary and Secondary eye care; Patient Safety and Quality of Care Improvements. This journal is indexed on PubMed
Dovepress

Central and CAS, and is the official journal of The Society of Clinical Ophthalmology (SCO). The manuscript management system is completely online and includes a very quick and fair peer-review system, which is all easy to use. Visit http://www.dovepress.com/ testimonials.php to read real quotes from published authors. 